\title{
Thickness dependences of optical constants for thin layers of some metals and semiconductors
}

\author{
S.A. Kovalenko, M.P. Lisitsa \\ Institute of Semiconductor Physics, NAS of Ukraine, Prospect Nauki 41, Kiev, 03028, Ukraine
}

\begin{abstract}
The review comprises investigations devoted to determination of refractive index and absorption coefficient dependences on thickness for thin films of metals and atomic semiconductors.

It has been shown that erroneous results were obtained in many papers and correct interpretation of the latter is absent. The reason that breaked the solution of the problem of dimensional optical phenomena in thin layer physics was ascertained.
\end{abstract}

Keywords: thin films, refractive index and absorption coefficient, thickness.

Paper received 10.08.01; revised manuscript received 26.11.01; accepted for publication 12.12.01.

\section{Introduction}

Intensive investigations of zero-, one-, two-dimensional solid formations are directed on improving scientific basis of principally new electronic elements technology design which based on quantum dots, wires and wells. These researches have caused new stimulus for experimental and theoretical investigation of refractive and absorption indexes thickness dependences for solids possessing all types chemical bonds - from metals to semiconductors and dielectrics.

As a matter of fact, a thin layer as a single quantum well with infinitely high potential barriers unlike quantum dots and especially wires was studied widely in the first half of the $19^{\text {th }}$ century yet.

If we consider such optical parameters as refractive index $n$ and absorption coefficient $k$, to determination of which our work is devoted, then it is necessary to note that measurements of light intensities reflected and transmitted by the thin layer is the first stage in the basis of optical experimental methods. The second stage deals with calculation of $n$ and $k$ using equations received by different authors with various grades of restrictions. At the absence of computer technic it was impossible to process experimental data using exact equations.

In his time, considering the Fresnel formulae Stretton could not hold from the remark: "Complication, one would think of simple task, is amazing indeed. Formulae are too complicated to be understood at the first sight. The character of reflection phenomenon can be ascertained only from analyses of some boundary cases".

Above, the matter was about light reflection when the only interface surface operates between two media. If the participation of the second interface, that is surface between thin layer and substrate, or besides the free surface of the substrate, are necessary to be taken into account, we get too complicated relations for energy coefficients of reflection $R$ and transmittance $T$ for the layer-substrate system.

Below, it will be shown using examples of $\mathrm{Au}, \mathrm{Ag}$ and atomic semiconductors to what unpredictable results the calculation on the base of simplified relations can lead. Besides, new data concerning $n$ and $k$ thickness dependences for $\mathrm{Au}, \mathrm{Ag}, \mathrm{Se}, \mathrm{Te}, \mathrm{Ge}$ and $\mathrm{Si}$ thin films will be introduced. 


\section{S.A. Kovalenko et al.: Thickness dependences of optical constants for ...}

\section{2. $n$ and $k$ thickness dependences}

\subsection{Metal films}

It is well known that mass density correlates quite well with an optical density. It is confirmed, for example, by the fact that refractive index increase with growth of specific qravity for various sorts of optical glasses. At the formation wedgelike thin - layer samples of solids the specimen is formed automaticaly, material density of which falls to zero at transfer from continuous to island layer and complete disappearance of islands. The similar monotonous decrease should be characteristic for refractive index with decreasing thickness.

B. Pogany and I. Kraumkramer [1,2] were the firsts who have obtained the dependences of refractive index and absorption coefficient on thickness (Fig. 1). Curve 2 shows monotonous increase of the effective refractive index with decreasing thickness. Curve 1 possesses the sufficiently washed maximum that corresponds to the effective thickness $60 \mathrm{~A}$, and refractive index sharply increase to the value $n=3.6$ beginning from $d=20 \AA$ while in the second paper the refractive index exceeds the value 4 at $d=20 \AA$.

The difference in $n$ values seen from these two papers for all values of thickness is caused first of all by different

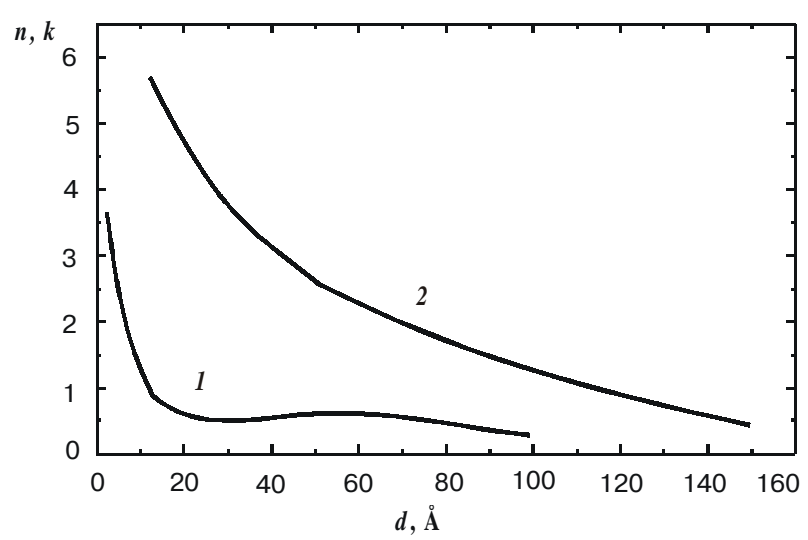

Fig. 1. Dependence of gold thin layer refractive index from thickness, where 1 the curve from scientific paper [1], 2 the curve from paper [2].

technological conditions of thin gold layer preparation. The common feature of two cited papers consist in catastrophical non-motivated growth of $n$ at $d \rightarrow 0$.

Four $n$ and $k$ thickness dependences of platinum, palladium, silver and copper for various light wavelength within the visible range of spectrum $[3$, p. $311 ; 4]$ are shown in Fig. 2. In a comparatively thick layer, $n$ and $k$ change a
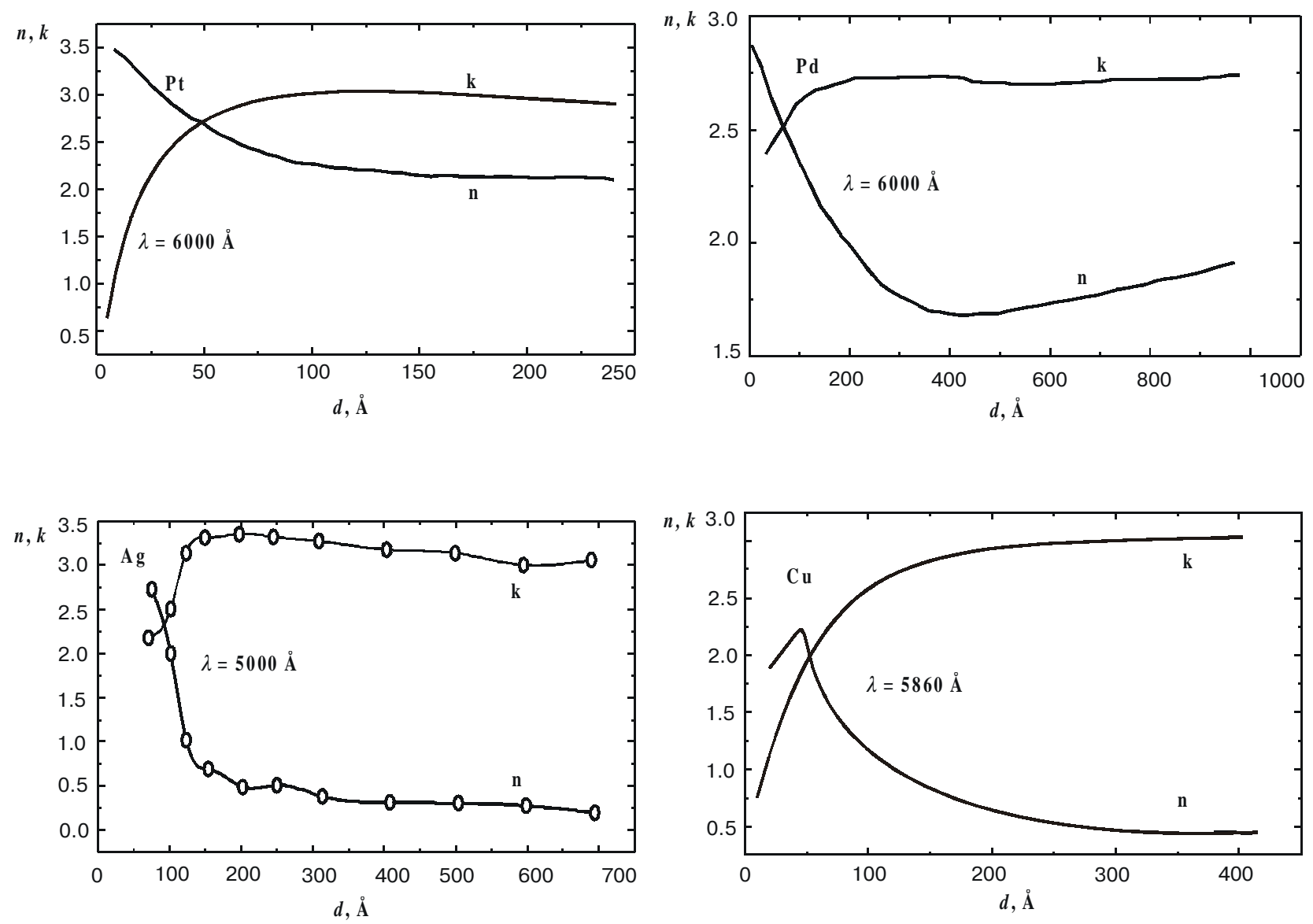

Fig. 2. Thickness dependence of $n$ and $k$ thin films

(a) platinum ([3], p. 311), (b) palladium ([3], p. 311), (c) silver ([4], p. 82), (d) copper ([3], p. 311) 


\section{S.A. Kovalenko et al.: Thickness dependences of optical constants for ...}

little. Quite another behaviour of $n$ is displayed at small thicknesses: the refractive index increases with decreasing thickness, similar to considered above gold films. The maximum is only observed for the copper layer with thickness $45 \AA$, although the general tendency of growing $n$ is kept. Refering to curves for silver, S. Methfessel wrote [4]: «Almost all performed measurements of optical constant dependences on thickness are qualitatively described by curves shown in the figure...». Sharp increase of refractive index with decreasing film thickness is not a subject of author's interest.

Note that similar dependences for various metals were received different authors in various laboratories at various time.

\subsection{Semiconductor films}

M.P. Lisitsa and M.G. Tsvelykh performed investigations of thickness dependences for thin layers of atomic semiconductors $\mathrm{Se}, \mathrm{Te}, \mathrm{Ge}, \mathrm{Si}[5,7,13]$ in the visible spectral range in 50-th years of the previous century. The spectral dependences of refraction indexes for Se, Te and Ge are shown in Fig. 3. As can be seen, $n$ increases when $d$ decreases. For selenium such curve is questionable since at $d=50 \AA, n=40$.

In these papers the intensities of three beams such as reflection from the side of layer, transmittion through layer and substrate, reflection from the substrate were measured.

Using the values of these intensities the values $n, k$ and $d$ were calculated on the base of the modern for that time $\mathrm{F}$. Abeles' method [8]. It is not necessary to measure the thickness of layer separately in accordance with this method. However, authors measured $d$ independently using exact method of equal chromatic order lines. It was revealed firstly that $d$ values for the same position do not coincide when measuring by two different methods. Secondly, $d$ values calculated on the base of F. Abeles' method were different for definite film area at various wavelengths. These results together with non-understanding increasing $n$ at decreasing $d$ was an obstacle for continuing these researches.

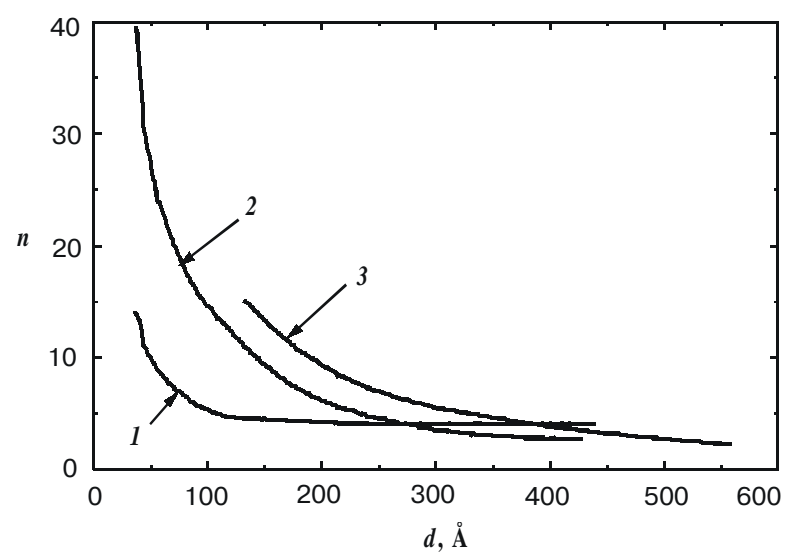

Fig. 3. Thickness dependence of refractive index for thin layer of atomic semiconductors, where 1 for Te at $\lambda=700 \mathrm{~nm}, 2$ for Se at $\lambda=540 \mathrm{~nm}, 3$ for Ge at $\lambda=720 \mathrm{~nm}$.

\section{The calculation of optical constants $n$ and $k$}

\subsection{Main formulae}

Usage of computer technic enables one to avoid restrictions which are typical for F. Abeles' equations [8] and use H. Murmann's equations [9] without simplifications. By the way, simplified formulae are used even last years, too [10, 11]. Below, we will analyse possibilities for calculation of $n$ and $k$ using exact formulae for photometric measurements in the case of normal incidence.

Our method for solution Murmann's equation is based on the determination of coordinates of the so-called "equal reflection and equal transmission curves crossing".

In common case, when the thickness of layer is known, the equations may be formally submitted as

$$
\left.\begin{array}{l}
R_{\text {exp }}=f(n, k), \\
T_{\exp }=\varphi(n, k) .
\end{array}\right\}
$$

The first equation characterizes the reflection coefficient of a thin layer-substrate system. The second one determines the transmission coefficient of such system. To solve this task, we will plot functions $f$ and $j$ in coordinates $n, k$. Changing value $k$ within limits $0<k<10$ in the visible region and $0<k<100$ in infrared ranges of spectrum, we can find corresponding $n$ values. In this case, the task is reduced to numerical solution of equations with one unknown quantity:

$$
\left.\begin{array}{l}
n_{R}=\psi(k) \\
n_{T}=\chi(k)
\end{array}\right\}
$$

The coordinates of crossing points for curves (2) are solutions of the system (1). To choose the correct solution, the following conditions should be used:

$n>k$ when $\lambda<\lambda_{p}$,

$n<k$ when $\lambda>\lambda_{p}$.

where $\lambda_{p}$ is a wavelength of thin layer substance bulk plasmon resonance. This value for gold equals to $385 \mathrm{~nm}$.

As example, plots of equal reflection and transmission curves for gold film with thickness $85 \AA$, which corresponds to the wavelength $\lambda=6400 \AA$, are shown in Fig. 4 . Taking into account the condition (3) the $n_{2}$ value should be considered as a true refraction index.

\subsection{Gold films}

The plots $\varepsilon_{1}(d)$ and $\varepsilon_{2}(d)$ are offen used in scientific papers instead of $n(d)$ what may be connected with difficulties of interpretation.

In paper [12] thickness dependences $\varepsilon_{1}(d)$ and $\varepsilon_{2}(d)$ for thin gold films were calculated using curves $R(d)$ and $T(d)$. Since in recent years this object lies within the 


\section{S.A. Kovalenko et al.: Thickness dependences of optical constants for ...}

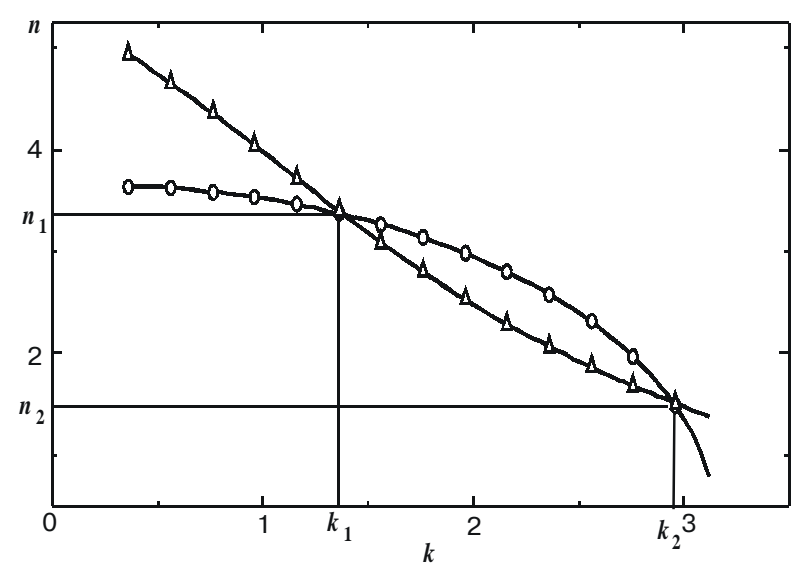

Fig. 4. The curve of equal reflection (1) and transmittion (2) for gold film by thickness $85 \AA$ at $\lambda=6400 \AA$. The refractive index of substrate equals to 1.458 .

sphere of our scientific interests, we have calculated $n, k$ thickness dependences using data [12] and well-known formulae;

$$
\begin{aligned}
& n=2^{-1 / 2}\left[\left(\varepsilon_{1}^{2}+\varepsilon_{2}^{2}\right)^{1 / 2}+\varepsilon_{1}\right]^{1 / 2} \\
& k=2^{-1 / 2}\left[\left(\varepsilon_{1}^{2}+\varepsilon_{2}^{2}\right)^{1 / 2}-\varepsilon_{1}\right]^{1 / 2}
\end{aligned}
$$

The results of the calculations are shown in Fig. 5.

Similar calculations have been fulfilled using H. Murmann's expressions.

Corresponding results for the set of two possible solutions are depicted in Figs 6 and 7. Taking into account condition (3), dependences shown in Fig. 7 should be considered as true ones.

Returning to Fig. 5, we observe full correspondence of plots by their shapes and numerical values to those shown in Figs 6, 7. However, the curves in Fig. 5 do not correspond to condition (3) because these were obtained by means combining two different solution parts.

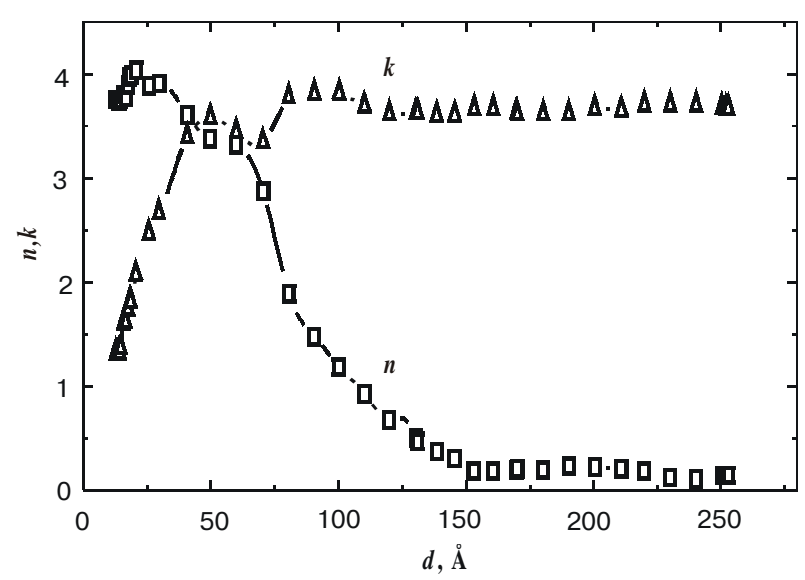

Fig. 5. Thickness dependences of $n$ and $k$ for gold layer calculated using the values $\varepsilon_{1}$ and $\varepsilon_{2}$ [12] for $\lambda=6328 \AA$.

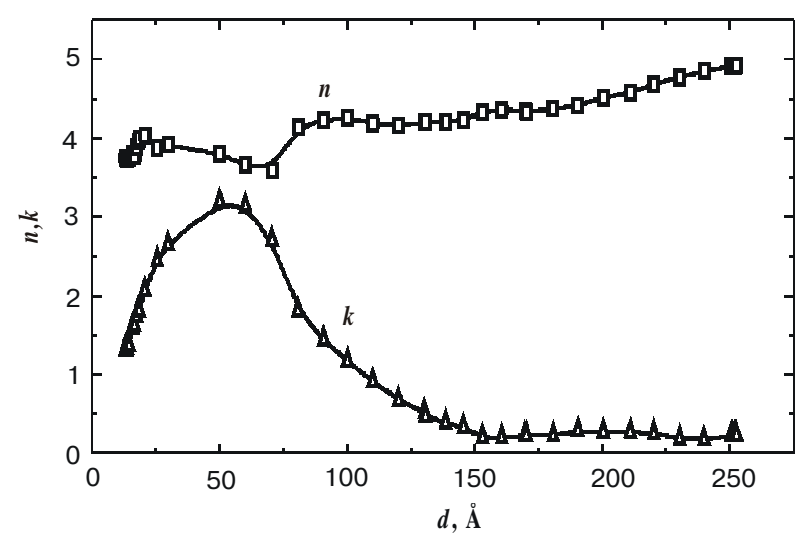

Fig. 6. Thickness dependences of $n$ and $k$ for thin gold film calculated on the base of Murmann's formulae (first solution) using data [12].

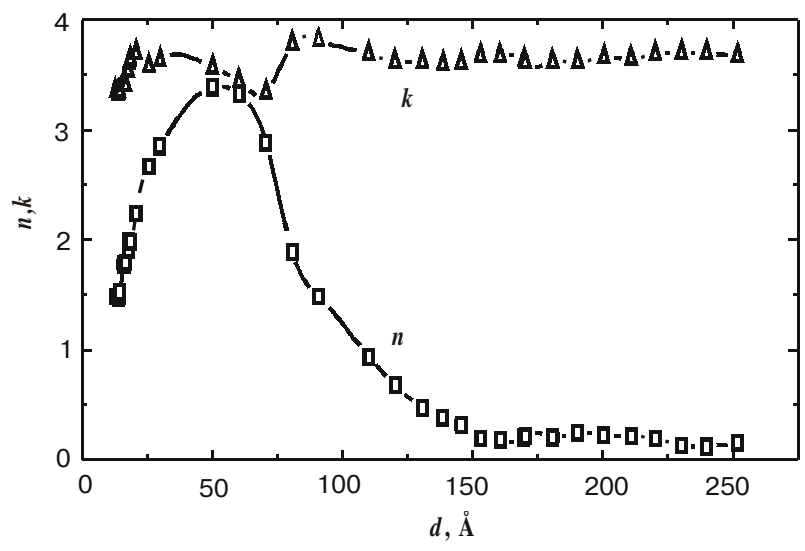

Fig. 7. Thichness dependence of $n$ and $k$ for thin layer of gold calculated on the base of Murmann`s formulae (second solution) using data [12].

\subsection{Silver films}

Applying the method of equal reflection and transmittion curves to silver films and using experimental $R(d)$ and $T(d)$ dependences [3, p. 276] we managed to get $n(d)$ and $k(d)$ plots that have numerical values close to real (Figs 8, 9). The curve in Fig. 9 possesses resonance maximum. The absorption index decrease with decreasing film thickness to the minimal value. Indisputably, the determination of $R$ and $T$ values from the published plots brings in significant mistake into the final result. However, reliability of the plot qualitative form is undoubtable.

Thus, it has been shown that it is necessary hold on the condition (3) for getting correct results.

\subsection{Films of atomic semiconductors \\ $\mathrm{Se}, \mathrm{Te}, \mathrm{Ge}, \mathrm{Si}$}

As it has been noted above, authors of publications [5-7] and, especially, [13] considered thickness dependences 


\section{S.A. Kovalenko et al.: Thickness dependences of optical constants for ...}

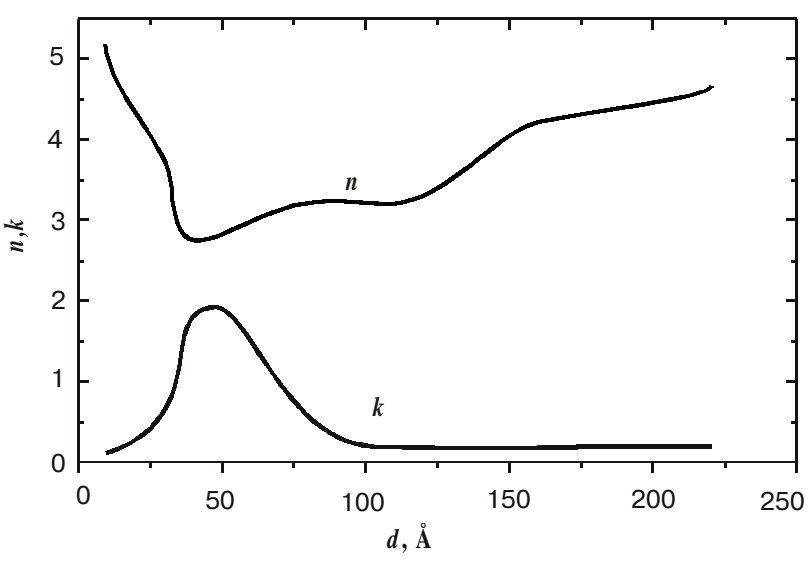

Fig. 8. Thickness' dependence of $n$ and $k$ for thin layer of silver calculated using data of paper [3, p. 276] (first decision).

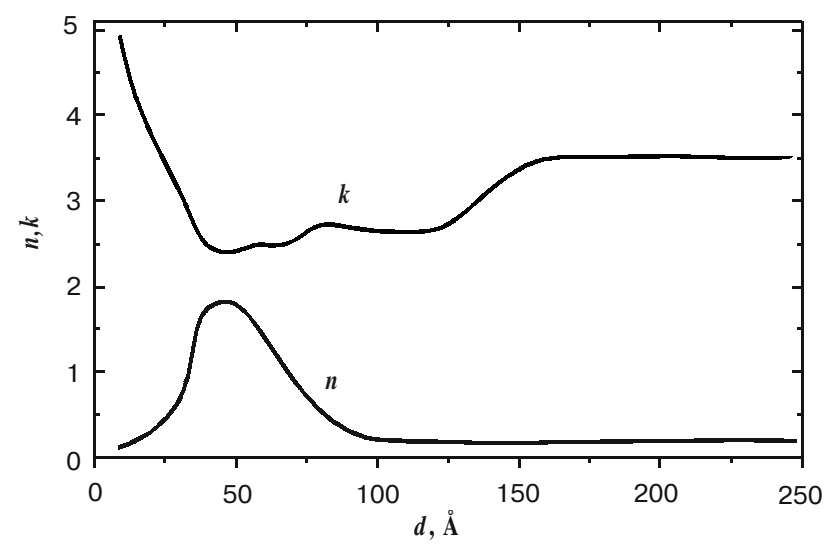

Fig. 9. Thickness' dependence of $n$ and $k$ for thin layer of silver calculated using data of paper [3, p. 276] (second decision).

of atomic semiconductors as insufficiently reliable. After 40 years from the moment of publications we have a possibility to return to experimental curves and use them to calculate $n(d), k(d)$ using new methods and computer technic.

New results are shown in Figs 10-13 for four kinds of atomic semiconductors. In all cases the thickness decreasing leads to decreasing a refractive index. However, for germanium, the refractive index increases beginning from $d=80 \AA$. Interval of refractive index increasing comprises only $30 \AA$, and the behaviour of refractive index at further thickness layer decreasing is unknown.

\section{Conclusions}

1. Using literature data it has been analysed the results of investigations that concern to dimensional phenomena in optics of thin layers. All attention has been concentrated of thin films of gold, silver, selenium, tellur, germanium and silicon. It has been shown that decreasing the refractive index with decreasing the thickness layer is mistaken.

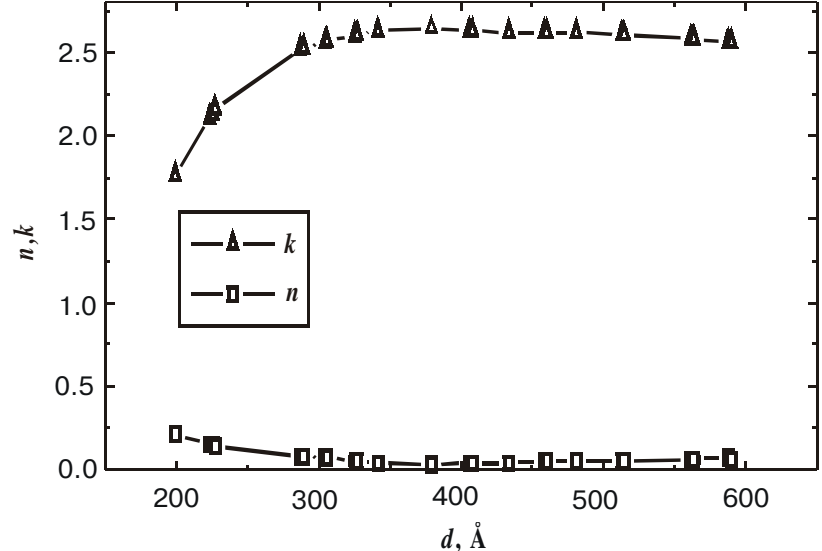

Fig. 10. Thickness' dependence of $n$ and $k$ for thin layer of selenium for $\lambda=700 \mathrm{~nm}$, calculated using data of scientific paper [6].

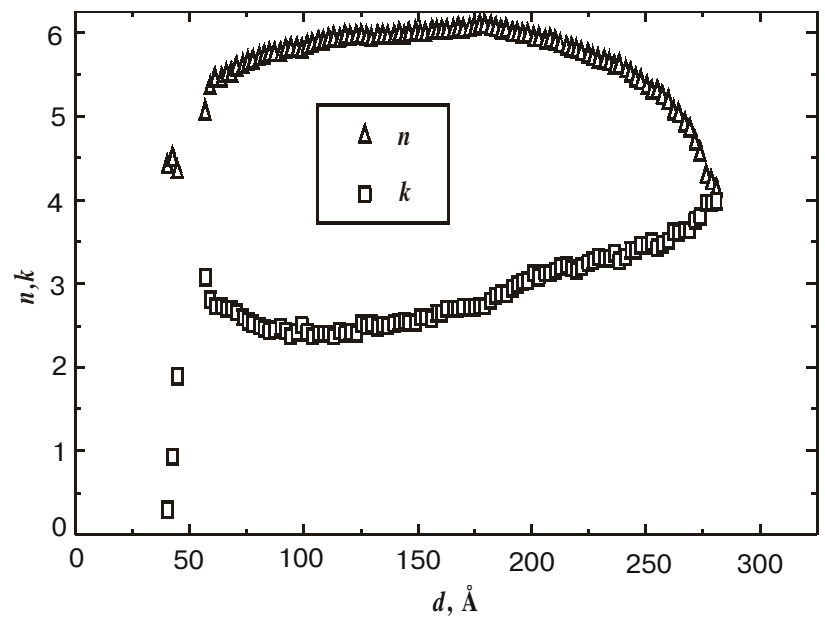

Fig. 11. Thickness' dependence of $n$ and $k$ for thin layer of tellure, calculated using data of scientific paper [5].

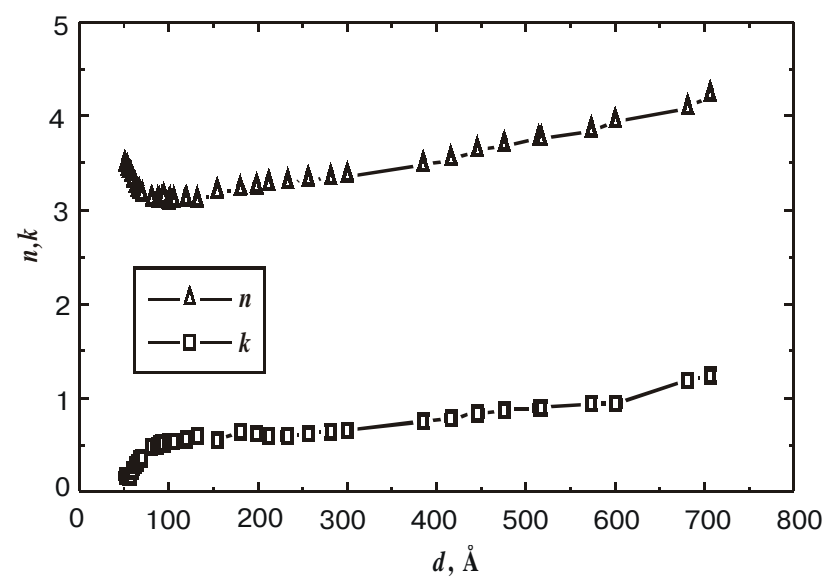

Fig. 12. Thickness' dependence of $n$ and $k$ for thin layer of germanium for $\lambda=750 \mathrm{~nm}$, calculated using data of scientific paper [7]. 


\section{S.A. Kovalenko et al.: Thickness dependences of optical constants for ...}

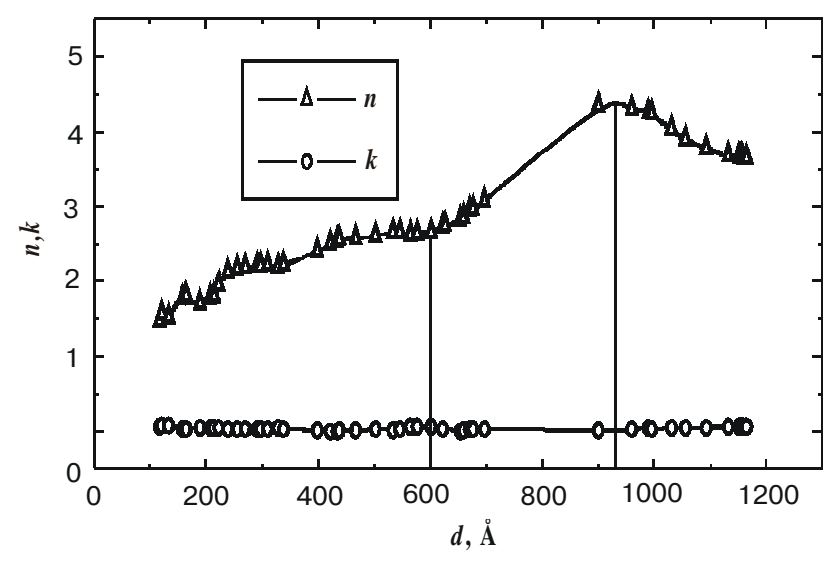

Fig. 13. Thickness dependence of $n$ and $k$ for thin a layer of silicon at $\lambda=760 \mathrm{~nm}$, using data of paper [14].

2. As been ascertained, the reason of errors in $n, k$ determination consisted in using rough mathematical approximations for optical constants calculation. Besides, ignoring the condition (3) led to mistaken choice of real value $n$ and $k$.

3. The plots from reviewed scientific papers $[4,5-$ $7,12,13]$ were used for new calculations of $\mathrm{Au}, \mathrm{Ag}, \mathrm{Se}, \mathrm{Te}$, Ge and Si thin film optical constants.

4. Despite significant experimental errors that appear when using curves from published papers, the right dependences $n(d)$ and $k(d)$ were calculated. The plots $n(d)$ presents dependences where the refractive index does not increase at $d \rightarrow 0$ but decreases.

\section{Reference}

1. B. Poqany // Ann. d. Phys. 49, p. 537 (1916).

2. I. Kraumkramer // Proc. Phys. Soc. 32, p. 537 (1938).

3. G. V. Rozenberg. Optics of thinlayer coatings, Gosenergoizdat. fiz.-mat. lit., ?oscow. 1958.

4. S. Methfessel. Dunne Schichten (Ihre Herstellung und Messung), p.68, Veb Wilhelm Knapp Verlag, Halle (Saale) (1953).

5. M. P. Lisitsa, N. G. Tsvelykh, Optics of thin film (II Te) // ?ptika i spektroskopiya, 4(3), pp.373-377 (1958) (in Russian).

6. M. P. Lisitsa, V. M. Mayevsky, N. G. Tsvelykh, Optics of thin film (III Se) // Optika i spektroskopiya, 5(2), pp.179-183 (1958) (in Russian).

7. M. P. Lisitsa, N. G. Tsvelykh, ?ptics of thin film (IV Ge) // Optika i spektroskopiya, 5(5), p.622 (1958) (in Russian).

8. F. Abeles // Rev. d Optique, 32, p. 257 (1953).

9. H. Murmann // Z. Phys., 80, p. 176 (1933).

10. I. Thomas, I. Pollini, G. Jezequel. Determination of the optical constants of absorbing isotropic materials from multiangle reflectance with polarized synchrotron radiation // JOSA, 5(3), pp. 344-350 (1988).

11. Zhuomin M. Zhang. Optical properties of a slightly absorbing film for oblique incidence // Appl. Opt. 38(1), pp. 205-207 (1999).

12. P. Gadenne. Etude de la Croissance des Couches Minces a Partir de Mesures Optiques et Electriques Pendant Leur Depot // Thin Solid Films. 42, pp. 369-381 (1977).

13. M. P. Lisitsa, N. G. Tsvelykh, Optics of thin film (V Si) // Optika i spektroskopiya 7(4), pp.533-557 (1959) (in Russian). 